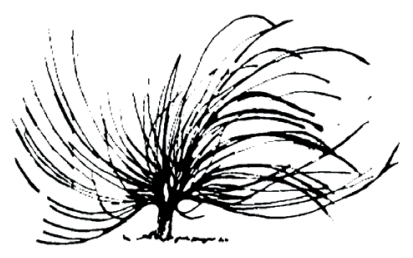

\title{
Caracterización social de la evaluación de los aprendizajes apoyada en entornos virtuales (autonomía, aprender a aprender y competencias), en la Escuela de Ciencias Exactas y Naturales (UNED)
}

\author{
Yency Calderón Badilla ${ }^{1}$ \\ Universidad Estatal a Distancia \\ Costa Rica \\ ycalderon@uned.ac.cr \\ Rosita Ulate Sánchez $z^{2}$ \\ Universidad Estatal a Distancia \\ Costa Rica \\ rulate@uned.ac.cr
}

\begin{abstract}
Resumen
Con el propósito de generar una caracterización social de la evaluación de los aprendizajes apoyada en entornos virtuales, en la Escuela de Ciencias Exactas y Naturales de la Universidad Estatal a Distancia en Costa Rica, se planteó una investigación con un enfoque cualitativo y comparativo de tres variables temáticas: la autonomía, la capacidad de aprender a aprender y el desarrollo de competencias de los estudiantes en su proceso de aprendizaje. Participaron treinta y cinco personas de cinco carreras, en sesiones de grupo y entrevistas a profundidad. Del análisis de las
\end{abstract}

Recibido: 20 de abril de 2019. Aprobado: 28 de octubre de 2019.

http://dx.doi.org/10.15359/rep.15-1.11

1 Profesora. Máster en Administración Educativa. Máster en Administración Universitaria. Máster en Evaluación Educativa de la Universidad de Costa Rica.

2 Profesora. Doctorado en Educación de Nova Southeastern University. Maestría en Administración de la Universidad de Costa Rica. 
categorías estudiadas, se observa la toma de decisiones de los estudiantes en la resolución y la organización de las tareas evaluativas, especialmente, para desarrollar capacidades de aprender más allá de su vida como aprendices. Igualmente, la mediación pedagógica fue uno de los aspectos de motivación más importantes para el estudiantado, aspecto por considerar en la planeación de los entornos virtuales. Por su parte, los participantes coincidieron en que sí se generan competencias intelectuales, sociales, personales y profesionales, como el uso de la tecnología, la capacidad de organización y planificación, la comunicación escrita, la gestión de la información, el compromiso con el aprendizaje y la generación de conocimientos para la sociedad.

Palabras clave: evaluación del aprendizaje, educación a distancia, entornos virtuales, implicaciones sociales.

\begin{abstract}
With the purpose of generating a social characterization of the evaluation of learning supported in virtual environments in the School of Exact and Natural Science of the Distance State University of Costa Rica, a research was proposed with a qualitative and comparative approach of three thematic variables: autonomy, the ability of learning to learn, and the development of competences of students in their learning process. Thirty-five people from five majors participated in group sessions and in-depth interviews. From the analysis of the categories studied, students' decision making is observed in the resolution and organization of assessment tasks, especially to develop abilities to learn beyond their life as students. Likewise, pedagogical mediation was one of the most important aspects of motivation for the students, an aspect to be considered in the planning of virtual environments. On the other hand, the participants agreed that intellectual, social, personal, and professional competences are generated, such as the use of technology, the capacity for organization and planning, written communication, information management, commitment to learning, and the generation of knowledge for society.
\end{abstract}

Keywords: evaluation of learning, distance education, virtual environments, social implications. 


\section{Introducción}

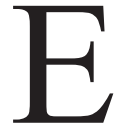

$\mathrm{n}$ estudios previos a lo interno de la Universidad Estatal a Distancia (UNED), se ha señalado la importancia de cumplir con lo establecido en el Modelo Pedagógico Institucional en las nuevas prácticas, en especial, con los campus virtuales de aprendizaje (Salas, 2011). Lo anterior, dado el cambio vertiginoso en las aulas universitarias, impulsado por las tecnologías de información y comunicación (TIC), (Corrales, 2005), al emerger nuevos espacios para implementar una evaluación de los aprendizajes, de manera sumativa y formativa. En este sentido, Fernández y Portillo (2009) sugieren la utilización de mecanismos de comunicación efectiva en la evaluación de los aprendizajes, como correos electrónicos, portales y páginas web, con el afán de proyectar conocimiento que sea transversal en la universidad y promover actividades de participación, como foros para docentes y estudiantes.

La Escuela de Ciencias Exactas y Naturales, como dependencia adscrita a la Vicerrectoría Académica, en la estructura organizacional de la UNED, comenzó a utilizar las plataformas de aprendizaje, a partir del 2001. Como parte de los procesos de autoevaluación, los programas de estudio propiciaron nuevas estrategias, técnicas e instrumentos de evaluación. Se abandonó la práctica tradicional de evaluar únicamente con pruebas escritas y se evolucionó en la evaluación de los aprendizajes mediada en las plataformas virtuales institucionales disponibles, con opciones de portafolios, foros académicos de participación obligatoria, pruebas en línea, rúbricas y listas de verificación (Calderón, 2006).

Es así como la Escuela de Ciencias Exactas y Naturales (ECEN) ha buscado que su quehacer académico se caracterice por poner en práctica los valores filosóficos propuestos en su plan estratégico (ECEN, 2007), de sensibilización y concientización en cuanto a equidad, compromiso, tolerancia, solidaridad y honestidad, enmarcados paralelamente en el modelo de educación a distancia.

\section{Marco teórico}

De acuerdo con De Alba (2011), en todo el entramado social existen relaciones de dominación específicas, de normalidad, de trastocar y transformar que no exoneran al ámbito educativo. Aunado a lo anterior, las universidades y sus actividades académicas también poseen dinámicas de cambio y transformación que tendrán impacto en 
la sociedad. Por lo tanto, según House (2000), en la responsabilidad social, las instituciones deben velar por la equidad, la justicia, la objetividad y la revisión de las políticas tanto institucionales y nacionales, de tal manera que no se contravenga al individuo ni al grupo.

De la misma forma, en la evaluación de los aprendizajes, hay presencia de aspectos relacionados con la generación del conocimiento, el acceso a la información, la democratización de la educación y la valoración de programas e instituciones, todos en una dimensión social. Desde esa perspectiva, se vinculan la autonomía, la capacidad de aprender a aprender y el desarrollo de competencias de los estudiantes en su proceso de aprendizaje.

\section{Consideraciones sociales de la educación}

Por una parte, en la Declaración Mundial sobre Educación Superior en el Siglo XXI (UNESCO, 1998), se resalta la importancia de la participación de todas las personas protagonistas, universitarias y sociales, en la toma de decisiones del proceso de evaluación institucional, pero en su particularidad institucional, diversidad y ambiente sociocultural. Por otra parte, el Informe Delors (1996) señala la necesidad de establecer dispositivos objetivos y públicos, para que los ciudadanos estén en condiciones de comprender el contexto y la situación del sistema educativo, así como su impacto sobre la sociedad.

Parte de los compromisos de una institución de enseñanza superior se relaciona con la gestión del conocimiento, el componente social, la búsqueda de la mejora y el ejercicio de la profesión desde un sentido ético. Aunado a lo anterior, la calidad universitaria, a través de los procesos de mejora, abarca la rigurosidad metodológica en su función evaluadora, la devolución de sus resultados y la implementación de acciones de mejora.

\section{Consideraciones sociales en la evaluación de los aprendizajes}

Mateo (2006) destaca el papel de la evaluación de los aprendizajes en dos direcciones: a) la primera se concibe como un instrumento de control al servicio de una sociedad deseosa de conocer los objetivos a partir de los cuales se orienta la educación y el rendimiento, obtenido como la rendición de cuentas; y b) la segunda, de dirección, es, más bien, un instrumento de mejora y optimización del propio sistema y de sus resultados, entendido como calidad educativa. Por ello, Santos 
(2000) indica que la evaluación no es una responsabilidad aislada de un docente, sino un hecho social del cual es responsable toda la institución.

En este sentido, el tema de la evaluación de los aprendizajes en un entorno virtual debe ser analizado desde sus implicaciones sociales, ya que la cultura tecnológica se establece en consonancia con los roles y los valores conferidos por la sociedad a los diferentes medios, recursos y herramientas, los cuales incluyen el uso de computadoras, Internet y plataformas virtuales de aprendizaje.

\section{Entornos virtuales y sus consideraciones sociales}

Algunos autores como Adello, Castellet, Pascual y Sigalés (1997) (según se citan en García et al., 2007) y Bautista, Borges y Forés (2008) plantean una definición tradicional del entorno virtual de aprendizaje (EVA), al referirse a él como un espacio educativo que se alberga en la web y que requiere Internet para que se den interacciones sincrónicas o asincrónicas entre los participantes.

Bautista et al. (2008) resaltan los beneficios de la formación con uso de los entornos virtuales en línea, pues esto posibilita la participación de estudiantes de lugares alejados entre sí y ofrece ventajas a la universidad, al implementar cursos en línea. Se puede afirmar, entonces, el planteamiento de nuevas formas y relaciones sociales entre el estudiantado y la universidad, por parte de la incorporación de entornos virtuales de aprendizaje, al realizar la mediación pedagógica, planificar el currículo de enseñanza y evaluar los aprendizajes mediante otros medios tecnológicos de apoyo.

En este sentido, los cursos o las asignaturas en entornos virtuales reúnen una variedad de realidades heterogéneas y de procedencia sociolaboral diversa. De acuerdo con Bautista et al. (2008), la comunicación, el diseño y la acción docente requieren incorporar la contextualización de los contenidos. En otras palabras, es preciso que todos los participantes puedan adaptarse a las características y las necesidades individuales, mientras que se debe ser cuidadoso en cuanto a respetar las particularidades socioculturales representadas en el entorno virtual.

Morgan y O'Reilly (2002) sugieren que el diseño de la evaluación de los aprendizajes en entornos virtuales requiere hacerse desde la realidad de mundo, es decir, ser auténtica y a la vez abierta e inclusiva; en tanto se comprenda el contexto complejo del alumnado y se le brinden los apoyos para superar barreras personales y profesionales. 
Bautista et al. (2008) afirman que las condiciones personales y sociales de un estudiante pueden facilitar o limitar su desempeño en un entorno virtual de aprendizaje. Igualmente, señalan que el rol fundamental del docente en línea es ser acompañante del aprendizaje, un facilitador presente y accesible en el entorno virtual. Por lo tanto, la universidad y sus respectivas autoridades deben estar conscientes de las realidades y las capacidades del estudiantado, según su contexto social y cultural, así como de las competencias del personal docente. De tal manera, se pretende que el uso de entornos virtuales en la evaluación de los aprendizajes no sea una barrera o impedimento para la democratización de la educación superior.

\section{Consideraciones sociales de la evaluación de los aprendizajes en entornos virtuales}

Un aspecto por resaltar en cuanto a las consideraciones sociales es el tema de la autonomía en el estudiantado, de modo que se apropie de su proceso de enseñanza y aprendizaje. Al respecto, el Modelo Pedagógico de la UNED (2004) establece que la autonomía estudiantil:

Equivale a que el estudiante sea capaz de realizar aprendizajes significativos por sí solo en una amplia gama de situaciones y circunstancias. Debe darse importancia a la adquisición de estrategias cognitivas de exploración y descubrimiento, así como de planificación y regulación de la propia actividad (p. 17).

De acuerdo con lo anterior, la evaluación de los aprendizajes en entornos virtuales debe gozar de una diversidad de estrategias e instrumentos evaluativos que fomenten en el estudiante el sentido crítico e investigativo, lo cual lo lleve a interiorizar su aprendizaje y a resolver situaciones a partir de sus competencias y conocimientos.

Por su parte, según la Comisión para la Educación de la Unión Europea, el aprender a aprender es la "Capacidad para proseguir y persistir y organizar el propio aprendizaje, lo que conlleva realizar un control eficaz del tiempo y la información, individual y grupalmente" (Universidad Internacional de Valencia-UIV, 2014, p. 73). Por ello, la evaluación de los aprendizajes en entornos virtuales puede ser una herramienta que permita al estudiante tomar conciencia de su aprendizaje 
a corto, mediano y largo plazo, a la vez que pueda brindarle la posibilidad de identificar el error y superarlo de manera exitosa.

De acuerdo con el Modelo Pedagógico de la UNED (UNED, 2004), aprender a aprender es un principio irrenunciable de la educación a distancia. Además, al respecto indica:

Que el estudiante sea capaz de realizar aprendizajes significativos por sí solo en una amplia gama de situaciones y circunstancias. Debe darse importancia a la adquisición de estrategias cognitivas de exploración y descubrimiento, así como de planificación y regulación de la propia actividad (p. 24).

Con respecto al desarrollo de competencias, específicamente curriculares y laborales, Martínez (2007) indica que la sociedad reclama niveles superiores de profesionalismo, tanto en aquellos que tienen el compromiso de formar como en quienes que se están formando. Igualmente, manifiesta este autor que las instituciones educativas y las prácticas docentes deben contar con el reconocimiento social de las capacidades y las competencias técnicas que desarrollan en sus estudiantes.

\section{Metodología}

Este estudio formó parte de los objetivos específicos de la tesis de maestría denominada Valoración de las implicaciones éticas, sociales y didácticas de la evaluación de los aprendizajes apoyada en entornos virtuales (Calderón, 2017). Por lo tanto, se efectuó una investigación cualitativa, con un alcance exploratorio, para conocer las características sociales de la evaluación de los aprendizajes apoyada en los entornos virtuales, en la Escuela de Ciencias Exactas y Naturales (ECEN) de la Universidad Estatal a Distancia. Además, se indagó desde una perspectiva evaluativa, la cual, según Cook y Reichardt (2005), se utiliza cuando "es necesario sacar a la luz y tener en cuenta la multiplicidad de elementos y de valores que entran en conflicto en cualquier realidad social y a la cual el propio investigador no es ajeno" (p. 18).

Se construyeron tres categorías de análisis para determinar: a) promoción de la autonomía del estudiante, b) impulso de la capacidad de aprender a aprender y c) contribución en la generación de competencias intelectuales, sociales, personales y profesionales. 
Se escogieron ocho asignaturas (Análisis Real, Introducción a la Teoría de Números, Edafología, Manejo y Conservación de Suelos, Laboratorio de Química I, Química I, Metodología de la Enseñanza de las Ciencias y Matemática para Computación I) de cinco carreras de la ECEN (Enseñanza de la Matemática, Ingeniería Agronómica, Manejo de Recursos Naturales, Enseñanza de las Ciencias e Ingeniería Informática), para proceder con la selección de los participantes. Los criterios de inclusión de las asignaturas fueron: a) ser de un plan de estudios para el grado de bachillerato de la Escuela de Ciencias Exactas y Naturales, b) ser administrada por una cátedra de la ECEN, c) ofertarse en el primer cuatrimestre del 2014, d) contar con un nivel de virtualidad de intermedio a avanzado (UNED, 2017) y e) aplicar al menos dos instrumentos de evaluación en la plataforma de aprendizaje de la UNED.

Se contó con la participación a conveniencia del investigador y, por opción voluntaria, de quince estudiantes (siete mujeres y ocho hombres), quince docentes (seis mujeres y nueve hombres), con entre dos y cinco años de laborar en la UNED, y cinco personas encargadas de alguna de las cátedras de las asignaturas escogidas (todos hombres), todos dentro de un rango de edad de veinticinco a cuarenta y ocho años. Los estudiantes escogidos habían finalizado una de las ocho asignaturas seleccionadas del programa de bachillerato en la UNED y estaban matriculados en el primer cuatrimestre del 2014. Mientras, los docentes, estaban contratados e impartían una de las asignaturas seleccionadas en el primer cuatrimestre del 2014; asimismo atendían el entorno virtual en la plataforma de aprendizaje institucional. Participaron los encargados de cátedra (funcionarios administrativa y académicamente a cargo de las asignaturas y tanto el nombramiento como el seguimiento de los profesores, quienes administraban las asignaturas que utilizaban el entorno virtual como apoyo a la evaluación de los aprendizajes, durante el primer cuatrimestre del 2014, a la vez que esa asignatura estuvo ofertada en el periodo de estudio.

Para la recolección de la información, se efectuaron dos grupos focales con guías validadas por cuatro especialistas en el área educativa, entre ellos, un especialista con grado de maestría en administración educativa, un especialista con grado de maestría en tecnología educativa, una especialista con maestría en evaluación educativa y una doctora en educación con especialidad en metodología de la investigación y diseño instruccional. Las preguntas generadoras abarcaron las categorías 
de implicaciones sociales predeterminadas; la primera estuvo dirigida a estudiantes y la segunda a docentes. Igualmente, se realizaron entrevistas a profundidad con preguntas abiertas y dirigidas a los encargados de cátedra. Seguidamente, se transcribió y se analizó la información, mediante la construcción de matrices para el análisis de contenido, con una codificación de las menciones de los participantes, según las categorías y subcategorías antes descritas.

\section{Análisis de los resultados}

En este apartado, se presentan los principales hallazgos y su análisis, de acuerdo con cada una de las categorías de estudio establecidas y con la opinión expresada por cada grupo de participantes, estudiantes, docentes y encargados de cátedra. Para resguardar la identidad de los informantes, se hace referencia al código designado para cada uno de ellos en los comentarios que se transcriben, literalmente, de las opiniones expuestas. Los resultados se presentan acorde con las categorías del estudio.

\section{a. Promoción de la autonomía del estudiante y la evaluación de los aprendizajes apoyada en entornos virtuales}

En las manifestaciones expresadas por los estudiantes, se encontró coincidencias en cuanto a que el modelo a distancia promueve en ellos la responsabilidad y la organización en la entrega de las actividades de evaluación en los entornos virtuales. Por ejemplo, uno de los estudiantes participantes señaló: "La evaluación en entornos virtuales sí promueve la autonomía porque nosotros debemos ser organizados, responsables y establecer cómo y cuándo hacemos las tareas de plataforma" (GFE-3); mientras que otro estudiante exteriorizó: "en la mayoría de evaluaciones el estudiante aprende a no depender de otros compañeros, ya que su nota depende de sí mismo, y esto lo hace autónomo para buscar por sus medios la información necesaria para hacer las evaluaciones" (GFE-1).

Delolme y Müller (1986) mencionan que algunas características por asumir, por parte del estudiantado de modalidad a distancia, son "estudiar solo, recibir las instrucciones de manera distinta a la presencial, aprovechar al máximo los apoyos institucionales, asumir nuevas responsabilidades como ser docente de sí mismo, aprovechar los recursos y medios didácticos-tecnológicos orientadores de su proceso de aprendizaje" (p. 25). 
También el personal docente consultado relaciona el tema de la autonomía con la organización y el aprovechamiento. Por ejemplo, uno de los participantes explicó que: "el estudiante acomoda el estudio a su entorno diario, deberá aprender a distribuir su tiempo y hacerse responsable, esto es un gran logro, aprender a planificar, a estudiar y cumplir con tiempos" (GFD-3). En este mismo sentido, otro informante mencionó lo siguiente: "el alumno tiene un plazo y puede trabajar respetando su ritmo de aprendizaje" (GFD-4).

Los docentes participantes destacaron que el uso de plataformas o entornos interactivos facilita el cumplimiento de los objetivos y la transferencia de conocimientos e indicaron que "el estudiante va creando sus propios conocimientos a través de experiencias evaluativas (...) forman su propio criterio, independencia de trabajo, opiniones" (GFD-2).

Lo expresado por los docentes concuerda con lo mencionado por Esquivel (2009), en cuanto a que "el estudiantado deberá participar en la valoración de sus propios aprendizajes y en la valoración del aprendizaje de sus compañeros" (p. 133). En ese mismo sentido, el Plan de Desarrollo Académico de la UNED (2012) indica que: "el desarrollo de las estrategias de autorregulación del aprendizaje ha de ser objeto de especial atención (...) así como la evaluación entre pares, dado que el aprendizaje autónomo y auto gestionado exige hoy al estudiantado la habilidad de expresar su punto de vista, lo contraste con el de sus pares y negocie nuevos significados" (p. 42).

Los aspectos de organización son las apreciaciones más destacadas por las personas encargadas de cátedra, con respecto a la autonomía del estudiante y los entornos virtuales, ya que:

El estudiante puede regular su aprendizaje. A su vez cuenta con una comunicación sincrónica o asincrónica con sus compañeros o docente, lo cual tiene como beneficio que el estudiante puede organizarse para ingresar a la plataforma en el momento que pueda y realizar las actividades formativas que se le sugieran (ECT-1).

Sin embargo, también destacan que el uso de rúbricas colabora en la formación de la autonomía del estudiante, ya que puede verificar sus logros o errores. Al respecto, un docente afirma que la autonomía en la evaluación "se promueve mediante la utilización de instrumentos 
con criterios descritos en las rúbricas de evaluación y promoción de autocríticas en foros colaborativos y de opinión" (ECT-5), y que con esas reglas claras desde el principio "el evaluador se convierte en guía del proceso de autoaprendizaje y no en completa fuente de consulta y única forma de obtención de pensamiento" (ECT-5).

De acuerdo con el Modelo Pedagógico de la UNED (2004), la autonomía implica que el estudiante se enfrente por sí mismo a una serie de situaciones y circunstancias de aprendizaje significativo, las cuales le conduzcan a la planificación, el descubrimiento y la regulación de su propia actividad cognoscitiva. Esta definición es coherente con las argumentaciones dadas por los participantes, al destacar que la evaluación de los aprendizajes apoyada en entornos virtuales promueve la indagación, la organización y el compromiso personal en el quehacer académico, durante el proceso de aprendizaje.

\section{b. Impulso de la capacidad de aprender a aprender}

Los estudiantes participantes mencionan la importancia del diseño de los instrumentos y de las estrategias de evaluación (con audios, videos, actividades multimedia, entre otros), para lograr captar la atención y generar motivación. Por ejemplo, uno de ellos expresa que "depende de cómo se diseñe la evaluación, hay espacios interactivos; sin embargo, la realidad es que en muchos casos los entornos son aburridos" (GFE2). Además, comentan sobre sus diferentes estilos de aprendizaje, así, un estudiante indica:

A mí me gusta aprender escuchando, a veces me grabo y me escucho yo mismo para aprenderme la materia; sin embargo, cuando entro al entorno virtual "hay mucho silencio" estoy frente a una computadora resolviendo mi tarea o quiz o solo revisando qué hay que hacer en la nueva unidad (GFE-1).

Un componente inmerso en el aprender a aprender a través de la evaluación de los aprendizajes apoyada en entornos virtuales es la motivación. Tal y como lo señala el Modelo Pedagógico de la UNED (2004), la motivación en el adulto que inicia una experiencia de aprendizaje permite mejores condiciones para persistir en el proceso educativo. De acuerdo con García et al. (2018), la motivación está también relacionada con las competencias del personal docente a la hora de enseñar a 
través de entornos virtuales, para estimular la motivación y la cohesión grupal, así como para fomentar procesos de autorregulación.

Por su parte, el personal docente consultado manifiesta que, indistintamente del medio para realizar la evaluación de los aprendizajes, debe considerarse un proceso que lleve a producir un cambio en el estudiante, a partir de las habilidades obtenidas en el desarrollo de las asignaturas. En este sentido, un docente informante indica que "Se considera al aprendizaje más un proceso que un producto en sí mismo, pues el cambio generado en el educando es una consecuencia de las experiencias vividas durante el proceso de enseñanza-aprendizaje, tal que logra integrar el conocimiento con las habilidades adquiridas" (GFD-1).

Además, los docentes participantes destacan las posibilidades y la diversidad de recursos existentes actualmente, los cuales contribuyen a promover el aprendizaje continuo, ya que la información se obtiene por medio de audio, imagen, multimedios, entre otros. Lo anterior hace necesario aprovechar las herramientas virtuales para ver el proceso de enseñanza-aprendizaje, que incluya la evaluación formativa y sumativa.

Se nota una coincidencia entre los estudiantes y los docentes participantes con respecto al aprender a aprender, lo cual ya está implícito en la dinámica del estudiantado y aprovecha el entorno virtual para mejorar sus capacidades de estudio y de aprendizaje.

Por su parte, el personal docente exterioriza su parecer en cuanto a que la era digital permite el acceso a la información de manera más ágil y a que la evaluación de los aprendizajes apoyada en entornos virtuales se convierte en una experiencia más expedita e inmediata, tanto para cada docente como para cada estudiante. En este sentido, "antes en la UNED las actividades de evaluación consistían únicamente en rendir pruebas escritas presenciales y quince días después el estudiante sabía su calificación, ahora, realizan quices en línea y de manera automática e inmediata tienen sus resultados de calificación" (GFD-4). Por ello, "el entorno o plataforma ha facilitado su aprendizaje y de qué manera. Un entorno virtual intuitivo, fácil de utilizar, atractivo estéticamente y adecuadamente estructurado es una herramienta para facilitar al estudiante su estudio autodidacta (GFD-6).

Con respecto a los encargados de cátedra (funcionarios que tienen a cargo la administración académica de varios cursos y sus profesores), estos manifiestan que la evaluación apoyada en entornos virtuales puede facilitar en el estudiante el aprender a aprender porque 
"implica la búsqueda de un aprendizaje significativo, que tome en cuenta que el docente es un facilitador del aprendizaje y el estudiante debe de construirlo- (ECT-1)" y que:

Desde el momento en que se está inmerso en un entorno virtual la misma naturaleza digital y el continuo cambio diario hace que este proceso se torne muy dinámico y favorezca el desarrollo de capacidades de aprendizaje cognitivas constantes. El desarrollo de la conducta de autoestudio y deber de estar pendiente de un entorno no estático hace que el estudiante se vea casi obligado a asimilar nuevos conocimientos y mejorar sus capacidades de estudio y elementos de aprendizaje (ECT-5).

También, una de las opiniones dadas implica incorporar "la autoevaluación y la heteroevaluación, mediante la utilización adecuada de la plataforma virtual y de las posibilidades que ofrece para la realización de actividades académicas como chats, foros" (ECT-2).

Con respecto a la necesidad de potenciar la capacidad de aprender a aprender, desde la perspectiva del Modelo Pedagógico UNED (2004), el estudiantado debe ser capaz de realizar aprendizajes significativos por sí mismo, con ayuda de la planificación y la regulación de sus propias actividades de aprendizaje, las cuales son la autoevaluación, la heteroevaluación y la evaluación formativa. Sobre todo, se debe tener en cuenta que, tal y como lo indican Fallas y Trejos (2013), "el futuro de la tecnología educativa no debe centrarse en la tecnología, sino en el aprendizaje con la tecnología; igualmente, ambos insisten en la importancia de ser un medio creador de ambientes de aprendizaje más significativos" (p. 88). Consecuentemente, Andrés, Herrero y Calderón (2017) señalan cómo el uso de la tecnología debe ser coherente con el currículo oficial de la carrera y con cada una de sus asignaturas, para que la evaluación se convierta en una actividad crítica de aprendizaje y genere nuevo conocimiento.

La comunidad docente y estudiantil coincide en que las estrategias de evaluación de los aprendizajes en los entornos virtuales les permiten desarrollar el aprender a aprender durante la carrera universitaria y, en general, para toda la vida. 


\section{c. Contribución a la generación de competencias intelectuales, sociales, personales y profesionales}

Los estudiantes participantes emitieron los siguientes comentarios sobre la evaluación de los aprendizajes apoyada en entornos virtuales: "familiariza al estudiante al uso de la tecnología que es importante hoy en día en el ámbito laboral y social" (GFE-4) y "además, el estudiante debe asumir el reto de cómo resolver cada situación que se le presenta que en algunas ocasiones ni el mismo tutor puede resolver entonces debe asumir el reto de poder solucionarlo" (GFE-2).

Lo anterior coincide con la visión de evaluar para aprender establecida en el Plan de Desarrollo Académico de la UNED 2012-2017 (UNED, 2012), en sus páginas preliminares, en las cuales se indica lo siguiente:

La evaluación de los aprendizajes en la educación a distancia no solo es utilizada para certificar los logros obtenidos por cada estudiante, sino que implica la evaluación continua, formativa y formadora, como momentos articulados en la estrategia metodológica. Lo relevante es quien aprende y la meta es el aprendizaje (p. 5).

Por su parte, los docentes consultados mencionan que la evaluación de los aprendizajes apoyada en entornos virtuales:

Potencia el protagonismo del estudiante en cada una de sus actividades, asumiendo compromiso en el proceso de aprendizaje mediante el proceso participativo que fortalece el intercambio y contraste de opiniones, lo cual le imprime a cada joven autonomía, competencias nuevas y habilidades valiosas para su desempeño social y laboral (GFD-1).

En relación con el hallazgo anterior, a partir de un estudio realizado por Vargas y Torres (2018), se encontró cómo las plataformas virtuales pueden potenciar y favorecer el aprendizaje, pero no por sí mismas, sino cuando incluyen una mediación pedagógica adecuada que favorece, sobre todo, a la comunidad estudiantil trabajadora, por ofrecer diversificación de recursos y una serie de opciones para desarrollar su aprendizaje. Dado lo dicho, es muy importante insistir con el personal docente en cuanto a no utilizar la tecnología como un fin, sino como un medio. 
El personal encargado de cátedras destaca la importancia de utilizar adecuadamente la plataforma y de seleccionar las actividades evaluativas pertinentes para promover las competencias intelectuales, sociales, profesionales y personales. Otro participante mencionó que la evaluación de los aprendizajes, de alguna manera, presiona a la población estudiantil; lo que tiene como consecuencia el desarrollo de habilidades y competencias no necesariamente de carácter intelectual, sino personales, como los hábitos de estudio o valores, por ejemplo, el compromiso y el cumplimiento. Al respecto, no se encontró evidencia de estudios que coincidan con el hecho de que los entornos virtuales deben fomentar, en el proceso de enseñanza-aprendizaje, el aprendizaje colaborativo y valores en el estudiantado que propicien el compromiso social. Por ende, se recomienda estudiar este tema en futuras sistematizaciones y encontrar un eje común para trabajar.

Además, el personal encargado de cátedra mencionó que "la evaluación genera algún grado de presión para cumplir con las tareas y para ello hay que poner a trabajar no solo las habilidades intelectuales. Es todo de manera integral, una cosa lleva a la otra" (GFD-3) y así "genera elementos vitales de conocimiento de destrezas propias de cada estudiante debido a su naturaleza descriptiva de competencias" (ECT-5).

Un resumen presentado por uno de los participantes menciona que la evaluación de los aprendizajes apoyada en entornos virtuales fomenta:

1. Competencias intelectuales, al cumplir, ya que la evaluación y la concreción de objetivos bajo los procesos formativos-sumativos generan un rendimiento académico esperado y el estudiantado participa activamente, llegando a las metas planteadas mediante una herramienta virtual.

2. Competencias sociales, al generar una relación estudiantedocente, estudiante-estudiante, para el cumplimiento de la competencia intelectual; se aplican las habilidades y estrategias sociales y de aprendizaje, con miras al proceso de interacción como autocontrol, participación y resolución de conflictos.

3. Competencias personales, por medio de un rendimiento óptimo, tanto en la parte formativa como en la sumativa; aplicación de aptitudes personales que pueden ser puestas en práctica en el desarrollo y la actitud correcta tanto para enfrentar procesos como para determinar resultados. 
4. Competencias profesionales, al poner en práctica los conocimientos obtenidos en una vida de competencia laboral, en la cual el estudiante puede generalizar el aprendizaje adquirido y se da una evaluación más contextualizada de lo aprendido.

Por su parte, los encargados de cátedra indicaron que la evaluación de los aprendizajes apoyada en entornos virtuales puede reflejar el compromiso social, en tanto que se seleccionen adecuadamente las prácticas o las modalidades de evaluación. Dichas evaluaciones deben considerar valores o ejes transversales implícitos, como ambiente, respeto a la diversidad, entre otros. Las estrategias de evaluación pueden promover la criticidad y la innovación, tanto en la población estudiantil como en el equipo docente.

Finalmente, uno de los participantes expresó que:

La sociedad actual debe estar comprometida con la innovación propia del desarrollo acelerado de las tecnologías de la información y enseñanza, en este sentido la evaluación de los aprendizajes dentro de los entornos virtuales es un elemento que permite que tanto docentes como estudiantes tengan participación dentro de este gran cambio que se está presentando dentro de los modelos de educación a distancia actuales y es un claro ejemplo de adaptación de la sociedad a las nuevas exigencias en materia de educación moderna (ECT-5).

\section{Conclusiones}

Se puede afirmar que la incorporación de entornos virtuales plantea nuevas formas tecnológicas de apoyo y relaciones sociales entre el estudiantado y la universidad, en cuanto a la mediación pedagógica, la planificación del currículo de enseñanza y la evaluación de los aprendizajes.

\section{Promoción de la autonomía}

Se puede concluir que, a partir de la comunicación oportuna en los entornos virtuales y de los resultados de la evaluación, el estudiantado toma decisiones para resolver o mejorar las asignaciones o las tareas y organizar su sistema de estudio. Por eso, se puede decir que la evaluación de los aprendizajes apoyada en entornos virtuales, como parte del 
proceso de enseñanza-aprendizaje, puede convertirse en una estrategia adecuada para fomentar la transferencia y la generación de conocimientos, junto con la promoción de la responsabilidad y autoorganización como pilares en la promoción de la autonomía de los estudiantes.

\section{Impulso a la capacidad de aprender a aprender}

En la UNED, todos los medios y los recursos (la tutoría, el texto, la plataforma, la llamada telefónica, el correo electrónico, entre otros) que se ofrecen al estudiantado como apoyo en el proceso de aprendizaje son medulares en el desarrollo de capacidades para aprender a aprender. Especialmente, la motivación brindada por medio del uso de entornos virtuales permite diseñar y resolver estrategias evaluativas.

Se puede afirmar que aprender a aprender consiste en interiorizar que el aprendizaje es permanente y una responsabilidad social e individual. Por lo tanto, la evaluación de los aprendizajes en entornos virtuales contribuye como estrategia metodológica para orientar el desarrollo docente como facilitador y guía en el proceso de enseñanza y aprendizaje.

\section{Competencias intelectuales, sociales, personales y profesionales}

La evaluación apoyada en entornos virtuales sí genera competencias intelectuales, sociales, personales y profesionales, porque la familiarizan con el uso de la tecnología, la cual es importante en el ámbito laboral y social. Algunas de las competencias instrumentales fortalecidas con el uso de la evaluación de los aprendizajes apoyada en entornos virtuales son aquellas que hacen referencia a la capacidad de organización y planificación, comunicación escrita, conocimientos tecnológicos, gestión de la información y la toma de decisiones en el proceso de enseñanza-aprendizaje.

Las competencias sistémicas y personales, que fortalecen el aprendizaje autónomo mediante la evaluación de los aprendizajes apoyada en entornos virtuales, son la motivación y el acercamiento a la tecnología. Por ello, la evaluación de los aprendizajes en entornos virtuales motiva en la población estudiantil el desarrollo de habilidades y competencias no necesariamente de carácter intelectual, sino personales, como los hábitos de estudio o los valores, como el compromiso, el cumplimiento, el diálogo y la ética implícita durante las participaciones realizadas en el entorno virtual. 
La evaluación de los aprendizajes en entornos virtuales promueve en el estudiantado compromisos para generar y construir conocimiento, a partir de sus aprendizajes y experiencias, de manera que le sirva para aplicarlo en los diversos contextos donde se desenvuelve, no solo en la universidad.

\section{Dimensión social de la evaluación de los aprendizajes en en- tornos virtuales}

Se puede concluir que la evaluación de los aprendizajes ejerce una influencia directa en la función social de la universidad, como herramienta para verificar los niveles de logro estudiantil en áreas cognitivas y afectivas, así como en cuanto a destrezas, habilidades y competencias que llevan a la acreditación, hasta lograr la meta máxima de graduarse e integrarse al campo laboral.

La evaluación de los aprendizajes apoyada en los entornos virtuales tiene varias implicaciones sociales: a) autonomía (autorregulación, compromiso personal y con la sociedad) b) aprender a aprender (uso de información, habilidades tecnológicas, uso de ejes transversales e interacción docente-estudiante) y c) formación de competencias (motivación, transferencia y generación de conocimientos) (ver figura 1).

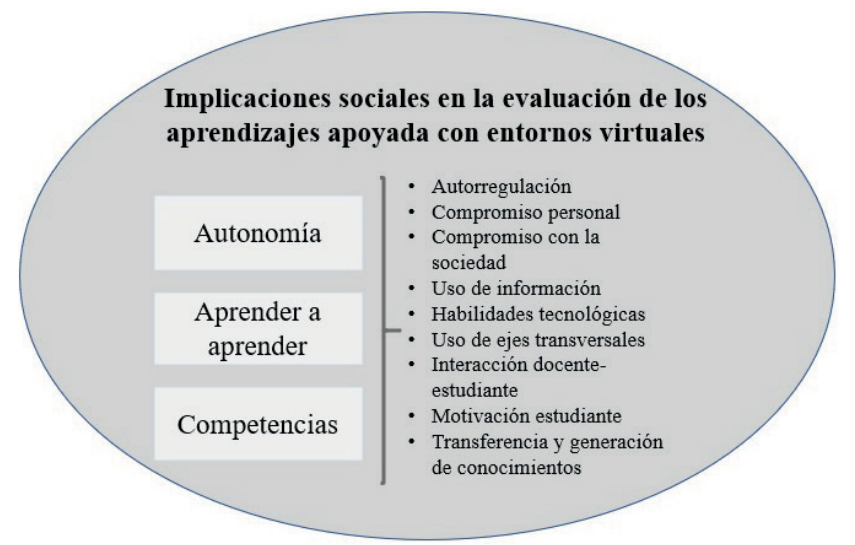

Figura 1. Implicaciones sociales de la evaluación de los aprendizajes apoyada en entornos virtuales desde la autonomía, el aprender a aprender y el desarrollo de competencias

Nota: Adaptado de Calderón (2017).

Desde los referentes teóricos, se puede concluir que la evaluación de los aprendizajes apoyada en entornos virtuales contribuye 
con el fortalecimiento de la autonomía estudiantil, cuando el equipo docente toma conciencia de la utilidad de la evaluación formadora y la implementa en el proceso de enseñanza-aprendizaje. Asimismo, la evaluación apoyada con entornos virtuales desarrolla nuevas competencias laborales y para la vida cotidiana del estudiantado, como la autorreflexión, el aprendizaje a partir de la identificación de los errores, acciones anticipatorias o preventivas, así como el desarrollo de competencias instrumentales, referidas a la capacidad de organización, planificación y toma de decisiones. Además, se consideran habilidades personales, como la interacción, el trabajo colaborativo y la creación de conocimiento de manera conjunta con sus pares, al igual que competencias sistémicas, como el liderazgo, la autonomía, entre otras.

Como aporte emergente se concluye que el compromiso personal de los docentes en su interacción con los estudiantes genera motivación, tanto en la transferencia de conocimientos de unos a otros como en la generación del saber en su formación profesional y, por ende, en el compromiso con la sociedad.

\section{Recomendaciones}

Para el desarrollo de la autonomía en el estudiantado, se recomienda trascender la evaluación sumativa e implementar tanto la coevaluación como la autoevaluación, pues tienen una función pedagógico-didáctica.

En cuanto al desarrollo de la capacidad de aprender a aprender, se debe promover la incorporación de diversos tipos de evaluación como la formadora, la formativa, la diagnóstica, la auténtica, entre otras, aprovechando las tecnologías de información y comunicación.

La mediación pedagógica en los entornos virtuales es la clave para desarrollar una asignatura de calidad, por lo que se recomienda cursos de capacitación y asesoría para el personal docente que implementen la evaluación de los aprendizajes en esos espacios tecnológicos, con la intencionalidad de que coincidan la metodología con los recursos $\mathrm{y}$ los medios educativos.

En el desarrollo de competencias, la evaluación se debe inculcar en la comunidad estudiantil, la cual debe asumir el reto de cómo resolver situaciones cotidianas con la ayuda de la tecnología.

No cabe duda de que el compromiso social es una responsabilidad compartida con el estudiantado y el grupo docente, el cual ha de 
considerar el uso de entornos virtuales como un canal para fortalecer valores y establecer diálogos a partir de temas transversales o de interés social.

Se destaca que el proceso de enseñanza-aprendizaje (el cual incluye la evaluación) es una estrategia por desarrollar de manera compartida y que repercute en el bienestar de muchos actores de la sociedad. Se afirma también, respecto a la creación del conocimiento, que esta debe ser compartida de forma tal que se promueva la autorrealización de los actores involucrados, para así fomentar el desarrollo de una sociedad más justa.

También se resalta, indistintamente del medio utilizado, el hecho de que, al evaluar el aprendizaje adquirido, siempre se incorpore la motivación, la interacción y el diálogo del equipo docente con el estudiantado, los cuales permitan formar personas para pensar y actuar de manera crítica, creativa y autónoma, con el propósito de que puedan desempeñarse en la sociedad de manera ética, profesional y comprometida con el bien común.

En cada plan de estudio y diseño curricular de las asignaturas, se debe incorporar los ejes transversales de la UNED; de esta forma, se une la evaluación con el compromiso social. Igualmente, las prácticas evaluativas pueden abordar el tema de ambiente, derechos humanos, respeto a la diversidad, entre otros. De esa forma, la evaluación en una plataforma debe asumir el compromiso social implícito en los ejes transversales, los cuales son prioritarios para una formación integral de las personas.

Los docentes, en el momento de planificar la evaluación de los aprendizajes, requieren seleccionar las técnicas e instrumentos a partir del currículo oficial de la asignatura, considerando que estos responden a una forma de enseñar y aprender, a distancia. Finalmente, dada la naturaleza exploratoria de este estudio, se recomienda realizar más investigaciones en este tema. 


\section{Referencias}

Andrés, C.; Herrero, D. y Calderón, Y. (2017). Estrategias de evaluación en una universidad a distancia: ¿alternativas o complementarias al examen escrito? Cuadernos de Investigación, 9(1), 157-164.

Bautista, G.; Borges, F. y Forés, A. (2008). Didáctica universitaria en entornos virtuales de enseñanza-aprendizaje (2. ${ }^{\mathrm{a}}$ ed.). Madrid: Ediciones NARCEA-Universitaria.

Calderón, Y. (2017). Valoración de las implicaciones éticas, sociales y didácticas de la evaluación de los aprendizajes apoyada en entornos virtuales. (Tesis de Maestría Académica en Evaluación Educativa, no publicada). Universidad de Costa Rica, San José, Costa Rica.

Calderón, Y. (2006). Informe anual de labores: Unidad de Asesoría Académica. Costa Rica: UNED, Escuela de Ciencias Exactas y Naturales.

Cook, T. D. y Reichardt, Ch. S. (2005). Métodos cualitativos y cuantitativos en investigación evaluativa (5. ${ }^{\mathrm{a}}$ ed.). Madrid: Morata.

Corrales, M. (2005). Estrategias de aprendizaje en línea: Un modelo teórico emergente en estudiantes de posgrados y universidades virtuales en español. (Tesis doctoral, no publicada). Instituto Tecnológico y de Estudios Superiores de Monterrey, México.

De Alba, A. y Martínez, M. (2011). Teoría y Educación: pensar con Foucault, nuevos horizontes e imaginarios en educación (Colección Educación Debates e Imaginario Social). México: UAZ.

Delolme, S. y Muller, A. (1986). El sistema de enseñanza-aprendizaje en la UNED. San José, Costa Rica: EUNED.

Delors, J. (1996). La educación encierra un tesoro. Informe a la UNESCO de la Comisión Internacional sobre la Educación para el Siglo XXI. Recuperado de http://www.unesco.org/education/pdf/ DELORS S.PDF

Escuela de Ciencias Exactas y Naturales (ECEN). (2007). Plan Estratégico de la ECEN-UNED 2008-2012. Recuperado de http://www. uned.ac.cr/ecen/organizacion/64-plan-estrategico-20082012

Esquivel, J. M. (2009). Evaluación de los aprendizajes en el aula: una conceptualización renovada. En E. Martín y F. Martínez (coords.), Avancesydesafiosenlaevaluacióneducativa(pp.127-143).Madrid: Organización de Estados Centroamericanos. 
Fallas, I. y Trejos, I. (2013). Educación en la sociedad de la información y el conocimiento. San José, Costa Rica: EUNED.

Fernández, R. y Portillo, J. (2009). Uso de las redes académicas avanzadas para la gestión del conocimiento en las Instituciones de Educación Superior. (Tesis de maestría no publicada). Instituto Tecnológico de Costa Rica, Cartago, Costa Rica.

García, B.; Serrano, E.; Ceballos, S.; Cisneros-Cohernour, E.; Arroyo, G. y Díaz, Y. (2018). Las competencias docentes en entornos virtuales: un modelo para su evaluación. RIED. Revista Iberoamericana de Educación a Distancia, 21(1), 343-365.

García, L.; Ruiz, M. y Domínguez, D. (2007). De la educación a distancia a la educación virtual. Barcelona: Ariel.

House, E. (2000). Evaluación, ética y poder (3. ${ }^{\mathrm{a}}$ ed.). Madrid: Morata.

Martínez, M. (2007). Profesorado y otros profesionales de la educación: Análisis para un sistema escolar democrático. Madrid: Octaedro y Ministerio de Educación y Ciencia.

Mateo, J. (2006). La evaluación educativa, su práctica y otras metáforas. España: Universidad de Barcelona y Ed. Alfaomega-Horsori.

Morgan, Ch. y O'Relly, M. (2002). Assessing Open and Distance Learners. Recuperado de http://www.unesco.org/education/pdf/DELORS S.PDF

Salas, S. (2011). El caso del Modelo Pedagógico de la UNED y su aplicación en los cursos en línea. (Tesis de maestría, no publicada). Universidad de Costa Rica, San José, Costa Rica.

Santos, M. (2000). Sentido y finalidad de la evaluación de la Universidad. Recuperado de http://firgoa.usc.es/drupal/node/8935

UNED. (2017). Consideraciones para el diseño y oferta de asignaturas en línea. San José, Costa Rica: UNED.

UNED. (2004). Modelo Pedagógico. Recuperado de https://www.uned. ac.cr/academica/images/igesca/materiales/24.pdf

UNED. (2012). Plan de Desarrollo Académico de la UNED. Costa Rica: EUNED.

UNESCO. (1998). Declaración mundial sobre la educación superior en el siglo XXI: visión y acción y marco de acción prioritaria para el cambio y el desarrollo de la educación superior. Recuperado de www.unesco.org/education/educprog/wche/declaration_spa.htm 
Caracterización social de la evaluación de los aprendizajes apoyada en entornos virtuales (autonomía, aprender a aprender y competencias), en la Escuela de Ciencias Exactas y Naturales (UNED)

Universidad Internacional de Valencia-UIV. (2014). Aprender a aprender: una competencia básica para el aprendizaje permanente. Recuperado de http://www.viu.es/aprender-a-aprender-una-competencia-basicapara-el-aprendizaje-permanente/

Vargas, L. y Torres, G. (2018). El uso de plataformas virtuales y su impacto en el proceso de aprendizaje en las asignaturas de las carreras de Criminología y Ciencias Policiales, de la Universidad Estatal a Distancia de Costa Rica. Revista electrónica Educare, 22(1), 1-20. 
